УДК 519.673:621.313.323

05.00.00 Технические науки

\section{АЛГОРИТМ ПОЛУЧЕНИЯ \\ МАТЕМАТИЧЕСКИХ МОДЕЛЕЙ ЭЛЕКТРОМЕХАНИЧЕСКИХ УСТРОЙСТВ ${ }^{1}$}

Бронов Сергей Александрович доктор технических наук, профессор кафедры вычислительной техники РИНЦ SPIN-код: 4712-7380

Поваляев Василий Александрович старший преподаватель кафедры вычислительной техники

РИНЦ SPIN-код: 3854-3132

Авласко Павел Владимирович

старший преподаватель кафедры вычислительной техники

РИНЦ SPIN-код: 9808-3545

ФГАОУ ВО «Сибирский федеральный

университет», Красноярск, Россия

Предложен и описан алгоритм получения комплекса математических моделей электромеханических устройств, который может быть затем формализован и реализован программно. До последнего времени реализация этого алгоритма была возможна только при ручном выводе всех выражений, что существенно затрудняло работу исследователей. Появление таких программ, как Maple, Mathematica и MathCAD обеспечило возможность автоматизации процесса получения аналитических выкладок, которая позволяет рассчитывать любые режимы любых типов электромеханических устройств, снижает вероятность появления ошибок и дает возможность изменять принимаемые допущения и обозначения в разрабатываемых моделях. Выбор программы MathCAD (в сравнении с Maple и Mathmatica) может быть обоснован тем, что MathCAD имеет самый удобный интерфейс, позволяя не только выполнять математические выкладки, но и представлять полученные результаты в обычной математической нотации. Полученные в MathCAD аналитические результаты можно сразу включать в численные модели

Ключевые слова: МОДЕЛИРОВАНИЕ, МАТЕМАТИЧЕСКАЯ МОДЕЛЬ, АЛГОРИТМ, ЭЛЕКТРОМЕХАНИЧЕСКОЕ УСТРОЙСТВО
UDC 519.673:621.313.323

Technical sciences

\section{ALGORITHM FOR OBTAINING MATHEMATICAL MODELS OF ELECTROMECHANICAL DEVICES}

Bronov Sergey Alexandrovich Doctor of Engineering, professor of the Department of computer engineering RSCI SPIN code: $4712-7380$

Povalyaev Vasily Alexandrovich Senior Lecturer of the Department of computer engineering RSCI SPIN code: $3854-3132$

Avlasko Pavel Vladimirovich Senior Lecturer of the Department of computer engineering RSCI SPIN code: 9808-3545 Siberian federal university, Krasnoyarsk, Russia

An algorithm for obtaining a complex of mathematical models of electromechanical devices, which can then be formalized and implemented in software, is proposed and described. Until recently, the implementation of this algorithm was possible only with the manual derivation of all expressions, which significantly hampered the work of researchers. The appearance of such programs as Maple, Mathematica and MathCAD provided the possibility to automate the process of obtaining analytical calculations that allows calculating any modes of any types of electromechanical devices, reduces the likelihood of errors and allows you to change accepted assumptions and designations in the developed models. The choice of the MathCAD program (in comparison with Maple and Mathmatica) can be justified by the fact that MathCAD has the most convenient interface, allowing not only to perform mathematical calculations, but also to present the results in ordinary mathematical notation. The analytical results obtained in MathCAD can be immediately included in numerical models.

Keywords: MODELING, MATHEMATICAL MODEL, ALGORITHM, ELECTROMECHANICAL DEVICE

\title{
Doi: 10.21515/1990-4665-134-097
}

1 Работа выполнена при финансовой поддержке РФФИ в рамках научного проекта № 16-38-00487 мол_а 


\section{Введение}

Процесс проектирования исполнительных электроприводов для космических аппаратов [1] обязательно сопровождается моделированием и требует разработки математических моделей элементов электропривода, в том числе моделей электромеханических устройств (ЭМУ) [2, 3].

При разработке таких математических моделей для аналитических выкладок удобно использовать программу MathCAD, которая позволяет производить символьные вычисления. Программирование таких вычислений напоминает программирование для численных расчётов, но имеет некоторые особенности.

\section{Алгоритм получения комплекса математических моделей}

\section{электромеханических устройств}

В статье предложен и описан алгоритм для получения комплекса математических моделей ЭМУ (рисунок 1), который позволяет автоматизировать аналитические выкладки с помощью программы MathCAD. Тип описываемого ЭМУ оказывает слабое влияние на предложенный алгоритм и определяется с помощью исходных уравнений связи потокосцеплений с токами обмоток ЭМУ и уравнениями, описывающими электрическое равновесие. Затем данные исходные уравнения обрабатываются предложенным алгоритмом и в результате получаются математические модели, характерные только для рассматриваемого типа электромеханических устройств.

Работа алгоритма начинается с исходного математического описания, включающего в себя следующие составляющие:

1) уравнения, описывающие электрическое равновесие, которые составлены в соответствии со схемой замещения ЭМУ, т.е. с учетом схемы, по которой подключены обмотки; 


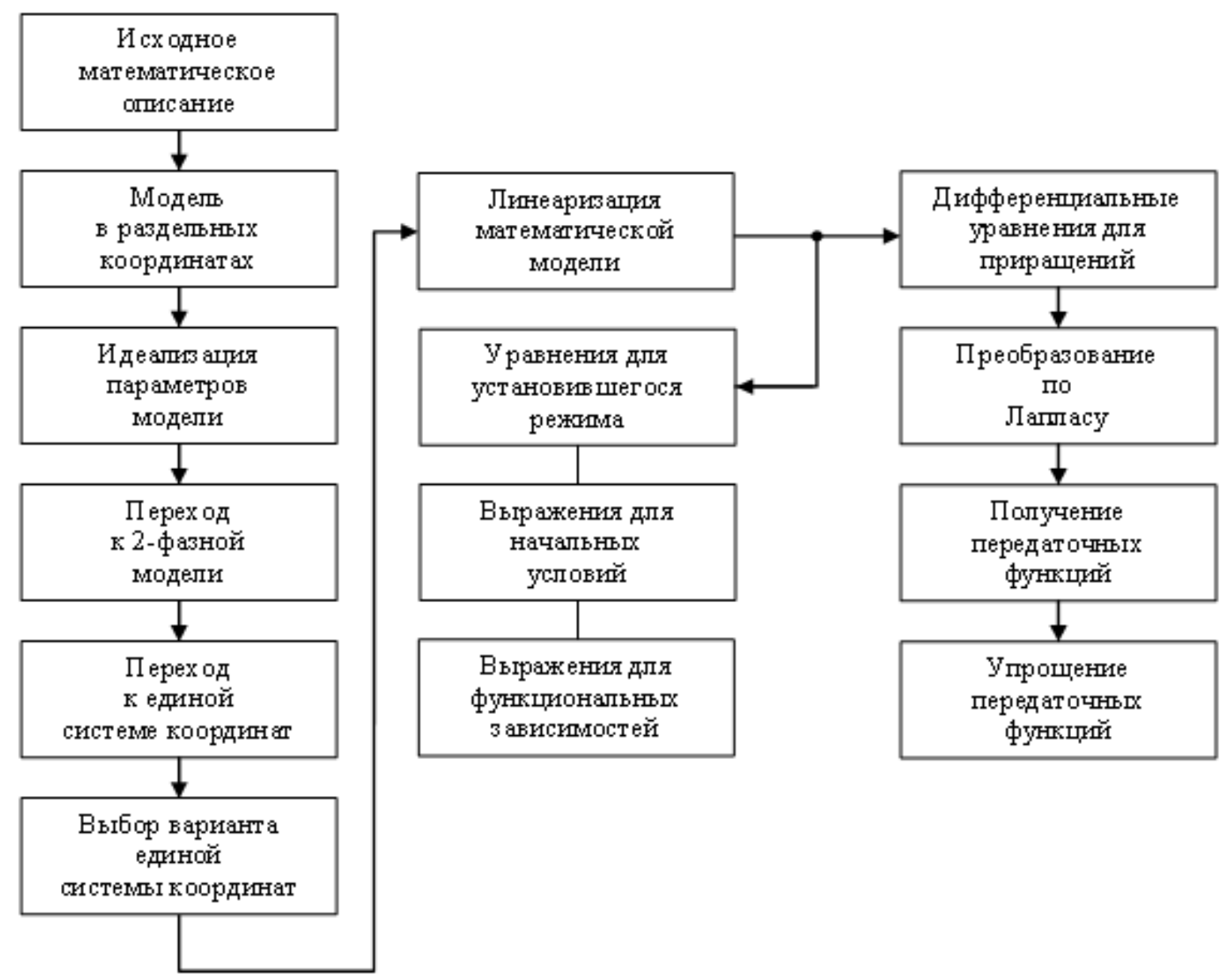

Рисунок 1 - Алгоритм получения комплекса

математических моделей электромеханического устройства

2) уравнения, описывающие связь между токами и потокосцеплениями ЭМУ;

3) выражения для расчета индуктивностей обмоток;

4) выражения для расчета электромагнитной энергии;

5) выражения для расчета электромагнитного момента.

Далее с помощью данного математического описания получают математические модели.

Математическое описание отличается от математических моделей своей степенью упорядоченности. Математическое описание представлено в виде набора уравнений и формул, которые в дальнейшем подставляются друг в друга разными способами. В итоге получаются математические 
модели, представляющие собой системы уравнений, в которых чётко выделены входные и выходные величины и параметры.

Получение модели в раздельных координатах обмоток происходит автоматически с помощью информации, полученной из схемы замещения и схемы, по которой размещены обмотки статора и ротора, а также применяемых к этим схемам методам анализа электрических цепей.

Эта модель включает в себя смешанные переменные состояния (токи и потокосцепления каждой обмотки). После дополнения модели уравнениями, которые описывают связи токов с потокосцеплениями, она используется для проведения расчётов переходных процессов в ЭМУ при помощи методов численного интегрирования.

Обычно смешанные переменные состояния не препятствуют применению модели в программах расчета с помощью численного интегрирования при условии, что такие программы не используют представление модели только в нормальной форме Коши, но допускают также промежуточные вычисления. Если потребуется представление модели ЭМУ в форме дифференциальных уравнений в нормальной форме Коши, то в данном случае осуществляется переход к однородным переменным состояния, содержащими только токи или только потокосцепления. Для этого токи выражаются через потокосцепления или потокосцепления выражаются через токи, и после этого происходит их подстановка в исходные уравнения.

В разрабатываемых математических моделях можно осуществлять учет различных неидеальности конкретного экземпляра ЭМУ, например, значения собственных индуктивностей и активных сопротивлений обмоток или некосинусоидальную зависимость взаимных индуктивностей различных систем обмоток для различных углов поворота ротора и т. д. Такие модели обычно используются для анализа переходных процессов с помощью методов численного интегрирования. 
В численном интегрировании в данном случае, обычно используются нулевые начальные условия, так как получить формулы для аналитического определения ненулевых начальных условий оказывается сложно ввиду большого количества тригонометрических функций (в установившемся режиме тригонометрическими являются напряжения питания, токи, потокосцепления, зависимости взаимных индуктивностей и некоторых собственных индуктивностей обмоток). Дополнительные сложности для расчёта начальных условий представляют неидеальности (например, разные значения сопротивлений и индуктивностей обмоток, не точно косинусоидальные зависимости взаимных индуктивностей от угла поворота и т.д.). При этом двигатель фактически не работает в установившемся режиме и его скорость всё время меняется (хотя, возможно, и незначительно).

Идеализация параметров модели осуществляется для дальнейших аналитических выкладок. К таковым относятся преобразования числа фаз и переход к единой системе координат обмоток. Преобразование числа фаз можно выполнять как с идеализированными, так и с неидеальными параметрами. Но необходимость в таком преобразовании появляется обычно на предварительном этапе перехода к единой системе координат обмоток. Это связано с переходом от 3-фазной модели к 2-фазной. Если оставить параметры неидеальными, то выражения получатся громоздкими. Они будут ещё более громоздкими, если далее выполнить преобразование системы координат. Поэтому перед процедурами преобразования числа фаз и координат выполняется идеализация параметров. В данном случае идеализацией является принятие допущений об электрической и геометрической симметрии машины, а также о косинусоидальном распределении магнитной индукции (т. е. о косинусоидальных зависимостях взаимных индуктивностей от угла поворота). 
Переход к двухфазной машине выполняется, если исходная машина является трёхфазной (и более). Это делается для минимизации уравнений и упрощения последующих аналитических выкладок. Иногда исходная модель трёхфазной машины имеет столько же уравнений, сколько и двухфазная (например, в случае соединения обмоток по схеме «звезда без нулевого провода»). В этом случае переход к двухфазной машине не приводит к уменьшению числа уравнений, но является подготовительным для последующего преобразования координат (перехода к единой системе координат обмоток).

Переход к единой системе координат обмоток выполняется с целью получения в последующем более простых аналитических выражений для установившегося режима и линеаризованной модели в приращениях. Преобразование координат приводит к тому, что в модели исчезают все синусоидальные выражения для переменных и параметров (взаимных индуктивностей), что существенно упрощает разложение всех величин в ряд Тейлора при линеаризации.

Выбор варианта единой системы координат обмоток выполняется в связи с тем, что в исходном виде преобразованная (единая) система координат повёрнута на произвольный угол (вращается с произвольной скоростью). Варианты системы координат могут быть разными, в частности, система координат может быть привязана: к статору (т. е. быть неподвижной в пространстве), к ротору (вращаться в пространстве с угловой скоростью ротора), к вектору питающего напряжения (вращаться с частотой питания), к любому другому обобщённому вектору (токов, потокосцеплений). При этом внешний вид модели меняется (какие-то переменные обращаются в нуль, какие-то остаются). Для разных типов электромеханических устройств и разных режимов работы бывают целесообразны различные частные варианты единой системы координат обмоток. 
Линеаризация математической модели выполняется с целью получения уравнений для установившегося режима и уравнений в приращениях. При этом используется разложение в ряд Тейлора с учётом только двух первых членов ряда - постоянной составляющей и приращения в первой степени.

Уравнения для установившегося режима получают из формул разложения в ряд Тейлора выделением постоянных составляющих в виде системы алгебраических уравнений.

Выражения для начальных условий необходимы, чтобы иметь возможность исследовать процессы в электромеханическом устройстве, начиная с любого момента, а не только с момента пуска, например, при торможении.

Выражения для функциональных зависимостей используются при расчёте зависимости угла нагрузки, к. п. д., токов и др. величин друг от друга в статическом (установившемся) режиме.

Дифференциальные уравнения для приращений получаются также из формул линеаризации и соответствуют малым отклонениям соответствующих величин от установившихся значений. На самом деле понятие «малые отклонения» оказывается различным для различных режимов работы. В некоторых случаях (например, для синхронного двигателя при постоянной частоте) малые отклонения от угловой скорости являются основным режимом работы. В других случаях, например, при реверсе асинхронного двигателя отклонения скорости, токов, потокосцеплений нельзя считать малыми. Дифференциальные уравнения в приращениях являются линеаризованной моделью электромеханического устройства, т. е. такая модель может использоваться в матричной форме, в форме передаточных функций, частотных характеристик не только для анализа, но и для синтеза замкнутых систем управления электроприводом. В исходном виде уравнения в приращениях записываются в матричной 
форме. Для использования их при синтезе применяются современные методы переменных состояния, например, связанные с распределением нулей и полюсов замкнутой системы.

Получение передаточных функций осуществляется на основе ранее полученных матричных дифференциальных уравнений в приращениях формальной заменой знака производной по времени на оператор Лапласа. После этого появляется возможность получения матричной передаточной функции (со многими входами и выходами).

\section{Заключение}

Вся указанная последовательность разработки математических моделей в том или ином виде используется в современной теории электрических машин. В данном случае она сведена в единый алгоритм, который может быть затем формализован и реализован программно. До последнего времени реализация этого алгоритма была возможна только при ручном выводе всех выражений, что существенно затрудняло работу исследователей. Появление таких программ, как Maple, Mathematica и MathCAD обеспечило возможность автоматизации процесса получения аналитических выкладок, которая позволяет рассчитывать любые режимы любых типов электромеханических устройств, снижает вероятность появления ошибок и дает возможность изменять принимаемые допущения и обозначения в разрабатываемых моделях. Выбор программы MathCAD (в сравнении с Maple и Mathmatica) может быть обоснован тем, что MathCAD имеет самый удобный интерфейс, позволяя не только выполнять математические выкладки, но и представлять полученные результаты в обычной математической нотации. Полученные в MathCAD аналитические результаты можно сразу включать в численные модели.

Работа выполнена при финансовой поддержке РФФИ в рамках научного проекта № 16-38-00487 мол_а. 


\section{Список литературы}

1. Бронов С.А., Авласко П.В., Марарескул А.В., Поваляев В.А. Проектирование электроприводов систем поворота антенн и батарей солнечных космических аппаратов // Авиакосмическое приборостроение. 2010. №2.

2. Копылов И.П. Математическое моделирование электрических машин. М.: Высшая школа, 2001.

3. Онищенко Г.Б., Локтева И.Л. Асинхронные вентильные каскады и двигатели двойного питания. М.: Энергия, 1979.

\section{References}

1. Bronov S.A., Avlasko P.V., Marareskul A.V., Povaljaev V.A. Proektirovanie jelektroprivodov sistem povorota antenn $\mathrm{i}$ batarej solnechnyh kosmicheskih apparatov [Designing of electric drives of systems of rotation of antennas and solar batteries of spacecrafts] // Aviakosmicheskoe priborostroenie [Aerospace instruments engineering]. 2010. №2.

2. Kopylov I.P. Matematicheskoe modelirovanie jelektricheskih mashin [Mathematical modelling of electrical machines]. M.: Vysshaja shkola [Moscow: Publishing House "High school"'], 2001.

3. Onishhenko G.B., Lokteva I.L. Asinhronnye ventil'nye kaskady i dvigateli dvojnogo pitanija [Asynchronous gated cascades and motors of double-way feed]. M.: Jenergija [Moscow: Publishing House "Energy"], 1979. 